\title{
Helicity generation and $\alpha$-effect by Tayler instability with z-dependent differential rotation
}

\author{
M. Gellert, G. Rüdiger, and D. Elstner
}

\begin{abstract}
Astrophysikalisches Institut Potsdam, An der Sternwarte 16, 14482 Potsdam, Germany
e-mail: [mgellert; gruediger; delstner]@aip.de
\end{abstract}

Received 3 May 2007 / Accepted 22 December 2007

ABSTRACT

\begin{abstract}
Aims. We investigate the instability of toroidal magnetic fields resulting from the action of $z$-dependent differential rotation on a given axial field $\boldsymbol{B}^{0}$ in a cylindrical enclosure where, in particular, the helicity of the resulting nonaxisymmetric flow is of interest. We probe the idea that helicity is related to the external field and the differential rotation as $\mathcal{H} \propto B_{i}^{0} B_{j}^{0} \Omega_{i, j}$.

Methods. We conduct isothermal magnetohydrodynamic simulations of a quasi-incompressible medium with finite viscosity and conductivity in a perfectly conducting container, and analyze both the kinematic and current helicity of the resulting field by regarding the nonaxisymmetric parts of the field as fluctuations.

Results. The observed instability leads to a nonaxisymmetric solution with dominating mode $m=1$. With the onset of instability, both kinematic and current helicity are produced which fulfill the suggested relation $\mathcal{H} \propto B_{i}^{0} B_{j}^{0} \Omega_{i, j}$. Obviously, differential rotation $\mathrm{d} \Omega / \mathrm{d} z$ only needs an axial field $B_{z}^{0}$ to produce significant helicity. Any regular time-dependency of the helicity could not be found. The resulting axial $\alpha$-effect $\alpha_{z z}$ is mainly due to the current helicity, the characteristic time scale between both the values is of the order of the rotation time. If the axial field is switched off, then the helicity and the $\alpha$-effect disappear, and a dynamo is not observed.
\end{abstract}

Key words. magnetohydrodynamics (MHD)

\section{Introduction}

Helicity in rotating turbulence plays a basic role in dynamo theory for the generation of large-scale cosmic magnetic fields (Krause \& Rädler 1980; Brandenburg \& Subramanian 2005). Usually stratification of density or turbulence itself is needed to generate helicity. We demonstrate that, even without stratification, helicity can exist due to the common action of magnetic fields and differential rotation. Whereas differential rotation depending on latitude is well-known from observations of star spots, differential rotation (of the interior of stars) depending on the $z$-coordinate only, appears more rarely. It is predicted and observable, for instance, in the tachocline of the sun (Rüdiger \& Kitchatinov 1997; Kitchatinov \& Rüdiger 2005), and influences the internal rotation of massive stars (Maeder \& Meynet 2003). It may open the possibility of studying nonaxisymmetric (large-scale) structures by shear-driven magnetohydrodynamic (MHD) instability of laminar flows (Braithwaite \& Spruit 2004; Braithwaite 2006).

Differential rotation transforms poloidal field components into toroidal components, which due to the Tayler instability (TI), become unstable if a critical amplitude is exceeded (Vandakurov 1972). Tayler (1973) showed that a magnetic field $B_{\phi}$ becomes unstable against nonaxisymmetric perturbations if the condition

$\frac{\mathrm{d}}{\mathrm{d} R}\left(R B_{\phi}^{2}\right)<0$,

where cylindrical coordinates $(R, \phi, z)$ are used, is violated. Constant fields or fields $B_{\phi} \propto R$, like those produced by $z$-dependent differential rotation from an axial field, are candidates for this instability. The most unstable Fourier mode for such a configuration is the nonaxisymmetric mode $m=1$ despite the smoothing action of the differential rotation. In a mean-field approach, due to the relation

$\alpha \simeq-\frac{\tau_{\text {corr }}}{3} \mathcal{H}$,

helicity $\mathcal{H}=\mathcal{H}_{\text {kin }}-\mathcal{H}_{\text {curr }}$ often indicates an existing $\alpha$-effect. $\mathcal{H}_{\text {kin }}$ and $\mathcal{H}_{\text {curr }}$ are the kinematic and current helicity,

$\mathcal{H}_{\text {kin }}=\left\langle\boldsymbol{u}^{\prime} \cdot \operatorname{rot} \boldsymbol{u}^{\prime}\right\rangle, \quad \mathcal{H}_{\text {curr }}=\frac{1}{\mu_{0} \rho}\left\langle\boldsymbol{B}^{\prime} \cdot \operatorname{rot} \boldsymbol{B}^{\prime}\right\rangle$,

respectively and $\tau_{\text {corr }}$ is the correlation time of the field pattern. Nonvanishing helicity could indeed be an indication of the existence of a resulting $\alpha$-effect on the basis of the TI. In case of the presence of an external field $\boldsymbol{B}^{0}$ and with a large magnetic Reynolds number, a dominating contribution from magneticfield fluctuations to the $\alpha$-effect can arise (Pouquet et al. 1976; Brandenburg \& Subramanian 2007).

Keeping in mind the pseudoscalar nature of helicity, a possible relation between helicity and both the external field and differential rotation would be

$\mathcal{H} \propto B_{i}^{0} B_{j}^{0} \Omega_{i, j}$

where $B_{i}^{0}$ means the external field components and $\Omega_{i, j}$ the gradient tensor of the basic rotation. The sign of $\mathcal{H}$ in Eq. (4) does not depend on the sign of the magnetic field, but it depends on the sign of the shear $\Omega_{i, j}$. Consequently, the dynamo number

$D=\frac{L^{2} \alpha \Omega}{\eta_{\mathrm{T}}^{2}}$ 


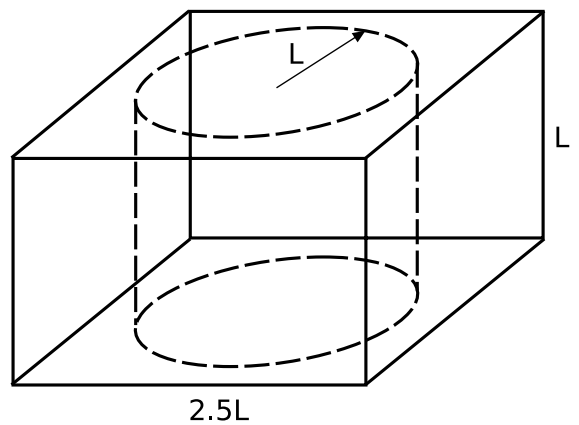

Fig. 1. The considered domain is a cylinder inside a box with Cartesian grid of side length $2.5 \mathrm{~L}$ and height $L$. The full set of equations is solved only in the cylindrical domain.

would now have a positive-definite sign. Here $L$ is the characteristic scale of the cylinder domain and $\eta_{\mathrm{T}} \simeq \tau_{\text {corr }}\left\langle u^{\prime 2}\right\rangle$. The sign of the dynamo number determines important properties of possible dynamo processes. E.g., a simple disk dynamo oscillates for a positive dynamo number and it is stationary for a negative dynamo number. Also, the magnetic Reynolds number of the $\alpha$-effect itself is important, that is, with Eq. (2) including the magnetic contribution,

$C_{\alpha}=\frac{L|\mathcal{H}|}{\left\langle u^{\prime 2}\right\rangle}$

( $\eta_{\mathrm{T}}$ does not include magnetic contributions, Vainshtein \& Kitchatinov 1983). We shall see that after the onset of TI, this quantity is of the order of unity when the averages are taken as an integral over the azimuthal direction.

In the following, the details of the model used are explained. Section 3 describes the onset of the TI in a cylindrical enclosure and the resulting field structure. In Sect. 4 , it is shown that - if the axial field is strong enough - the numerical simulations are in good agreement with relation 4 in the case of $z$-dependent differential rotation, where it simplifies to $\mathcal{H} \propto B_{z}^{0^{2}} \mathrm{~d} \Omega / \mathrm{d} z$ and only a (strong enough) $z$-component $B_{z}^{0}$ of the external field is needed. In Sect. 5 we show that the generated helicity is connected with an $\alpha$-effect, which is also proportional to the gradient of the differential rotation.

\section{Model}

We consider a differentially-rotating cylinder with radius $L$ and height $L$ embedded in a box with cartesian grid of side length $2.5 L$ and height $L$ (see Fig. 1). The cylinder radius is $20 \%$ less than the box side length, which appeared to be a good compromise between wasted computing power in the corner regions and decreasing influence from the geometry of the box. We calculated using the PENCIL code (Brandenburg \& Dobler 2002), a high-order finite difference code (sixth order in space and third order in time). Inside the cylinder domain, we solve both momentum and induction equations. Outside the cylinder, we kept the initial velocity to sustain the differential rotation via this no-slip conditions on the rim of the cylinder. On the top and the bottom, we apply stress-free conditions for the flow. For the magnetic field, perfect conductor boundary conditions are applied on all box boundaries. Additionally, in the region outside the cylinder, magnetic diffusivity $\eta$ is enhanced to a value ten times larger than inside the cylinder.

To keep the system nearly incompressible with a code for compressible media, the value of $\Omega(z)$ is chosen in a way that the resulting maximum of the (meridional) velocity never exceeds $16 \%$ of the speed of sound, which was set to $c_{\mathrm{S}}=8$. The initial density is set to $\varrho_{0}=1$, fluctuations are of the order of $10^{-4}$. The spatial resolution is $N=96 \times 96 \times 48$. Test runs with $N=128 \times 128 \times 96$ deliver only slight differences with smoother fields.

The initial magnetic field consists of the external timeindependent homogeneous field $\boldsymbol{B}^{0}=\left(0,0, B_{z}^{0}\right)$ applied only inside the cylinder. It is twisted into a strong toroidal field by the differential rotation. The strength of the generated field depends on $B_{z}^{0}$, varied between $B_{z}^{0}=0.01$ and $B_{z}^{0}=0.1$, and the gradient of the differential rotation $\mathrm{d} \Omega / \mathrm{d} z$, where the latter was fixed to $|\mathrm{d} \Omega / \mathrm{d} z|=1$ for all presented calculations. Based on the radius (set to $L=1)$ and the velocity at $z=1(U=\Omega L=1)$, and the viscosity $(v=0.03)$, the Reynolds number has a value of $\operatorname{Re}=33$. In the following text, system rotation always refers to the rotation on top of the cylinder and time is given in units of rotation time. The magnetic Prandtl number Pm is varied between $\mathrm{Pm}=10$ and $\mathrm{Pm}=30$ with no qualitatively change of the instability. In the following analysis, we concentrate on $\mathrm{Pm}=15$. We choose a large Prandtl number to encourage dynamo action, although we observed no dynamo.

\section{Instability}

If $B_{\phi}$ becomes strong enough, the TI occurs and leads to a growing nonaxisymmetric field. The largest nonaxisymmetric mode is $m=1$. By "strong enough" we mean that not only does the magnitude of $B_{\phi}$ reach high enough values, but also a certain threshold $B_{\phi} / B_{z}^{0}$ (with $B_{\phi} / B_{z}^{0}$ of the order of Pm) needs to be crossed, which is different from the case of pure toroidal fields (Tayler 1973; see Rüdiger et al. 2007). This means that an additional poloidal field component suppresses the instability. For Pm $=15$, the instability sets in at a Hartmann number of $\mathrm{Ha}=B^{*} R / \sqrt{\mu_{0} \varrho v \eta}=130$, where $B^{*} \approx 1$ means the maximal value of the generated toroidal field $B_{\phi}$. To produce such a strong toroidal component an external field $B_{z}^{0} \geq 0.04$ is needed. The instability did not occur for $B_{z}^{0}>0.08$.

The nonaxisymmetric structures appear first near the axis in the lower part of the cylinder where velocity magnitude is small, and grows to the steady state (Fig. 2). For $B_{R}$ and $B_{z}$, the $m=1$ mode becomes the largest one, in $B_{\phi}$ the axisymmetric mode remains dominant due to the permanently reproduced axisymmetric field. In the nonlinear regime, before the steady state is reached, higher modes also appear. Nonetheless, their magnitudes stay below $10 \%$ of the $m=1$ mode. One exception is the $m=4$ mode, caused by the box geometry. During the initial phase, this mode is already present and influences the magnetic field outside the cylinder near the box boundaries. However, the field inside the cylinder is much less affected, and the $m=4$ mode does not seem to influence the nature and onset of the instability at all. Additionally, during the growth phase of all other nonaxisymmetric modes, the $m=4$ remains nearly unchanged and does not play an extraordinary role in the final state. Modes higher than $m=4$ are not plotted in Fig. 2 .

The pattern of the $m=1$ mode (Fig. 3) possesses an azimuthal drift velocity relative to the system rotation. It rotates with 4.5 rotation periods of the cylinder, independent of the strength of the externally applied field. The TI in a TaylorCouette system exhibits the same characteristics of the unstable mode (Rüdiger et al. 2007). 


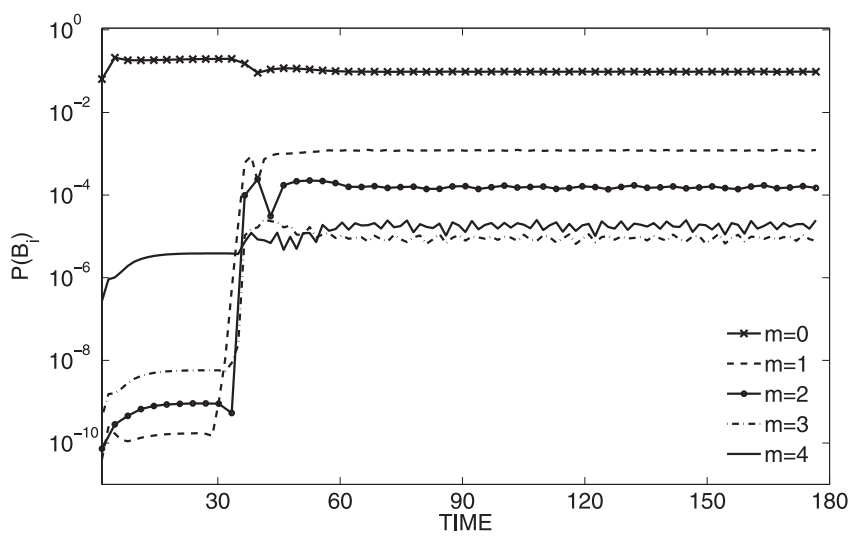

Fig. 2. The power spectrum of the Fourier modes of $B_{\phi}$ for $\mathrm{Pm}=15$ and $B_{z}^{0}=0.05$. Onset of the instability near $t=28$ where the dominating nonaxisymmetric mode $m=1$ starts to grow. Mode $m=4$ is nearly unaffected by the instability.

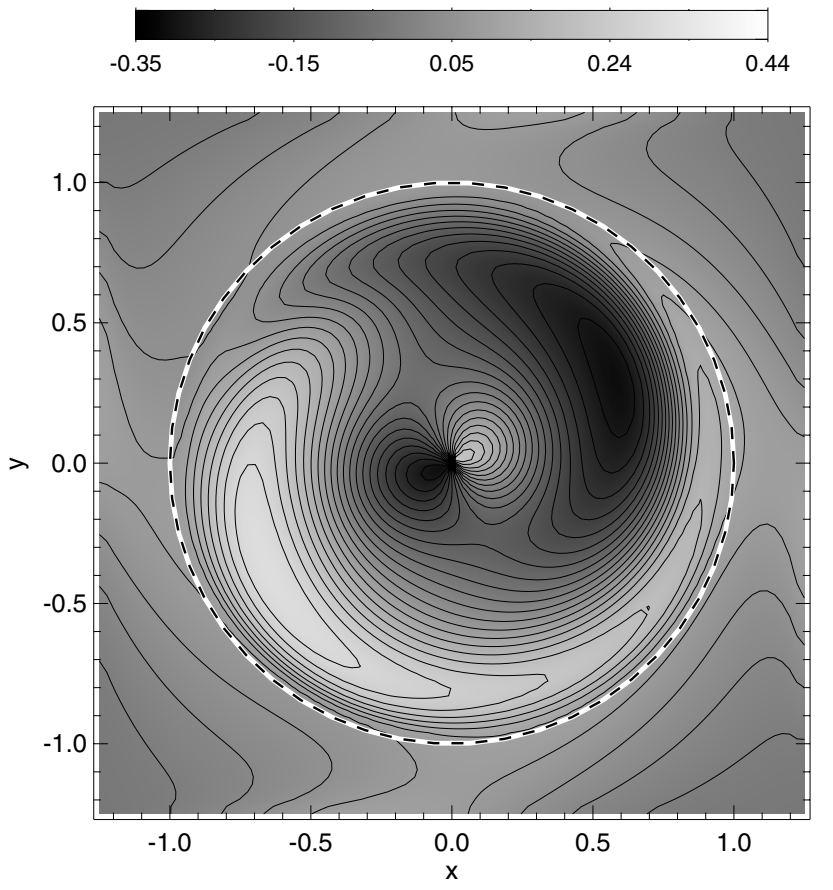

Fig. 3. The $m=1$ pattern in the magnetic field $B_{\phi}$ for $\mathrm{Pm}=15$ and $B_{z}^{0}=$ 0.05 . The pattern drifts relative to the systems rotation with 4.5 times the rotation period of the cylinder. The cylinder boundary is marked by the dashed black-white line.

\section{Generation of helicity}

To test the relation described by Eq. (4) in a simple setup, we restricted differential rotation to depend only on the $z$-direction ${ }^{1}$. For this case, the helicity $\mathcal{H}$ should depend on the sign of $\mathrm{d} \Omega / \mathrm{d} z$, but not on the sign of the external field and scale with the squared value of the latter, i.e. $\mathcal{H} \propto B_{z}^{0^{2}} \mathrm{~d} \Omega / \mathrm{d} z$. During the onset of the instability, the deviation of the flow structure from the original toroidal field is comparable to that of the magnetic field. Also, here the mode $m=1$ is the largest nonaxisymmetric mode and the higher modes appear with less energy.

1 The resulting non-conservative centrifugal force drives a meridional flow that, however, remains small (see Fig. 2).
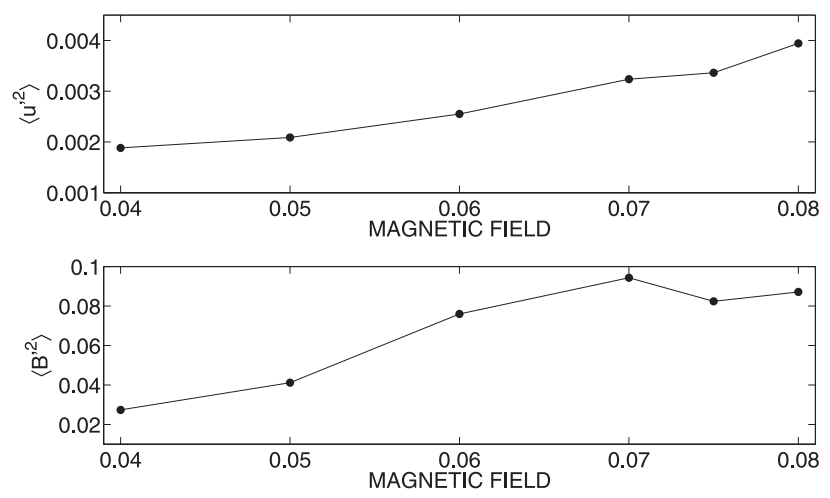

Fig. 4. The external field dependence on the energy of velocity and magnetic field fluctuations at $R=0.5$ averaged in vertical direction for $0.35<z<0.65$.

For the definition of fluctuations, we average velocity and magnetic field along the azimuthal direction, i.e.

$\langle\boldsymbol{u}\rangle=\frac{1}{2 \pi} \oint \boldsymbol{u} \mathrm{d} \phi, \quad\langle\boldsymbol{B}\rangle=\frac{1}{2 \pi} \oint \boldsymbol{B} \mathrm{d} \phi$

and regard the nonaxisymmetric parts as fluctuations of the fields. For constant $\mathrm{d} \Omega / \mathrm{d} z$, and with a homogeneous external field, the fluctuations should be constant in the vertical direction. Due to the finite height of the cylinder, boundary effects near the top and bottom induce additional flow disturbances and changes in the structure of the magnetic field in these regions. The independence is roughly preserved in the central region between $z=0.35$ and $z=0.65$. In Fig. 4 we show the energies of the fluctuations of velocity and magnetic field averaged over this central region, and their dependence on the external field $B_{z}^{0}$. The values are taken in radial direction at $R=0.5$. The magnetic energy always exceeds the kinetic energy. The ratio varies between 15 and 30. Thus, the instability is dominated by the magnetic field. This is also reflected in the helicities. We again use azimuthal-averaged quantities,

$\mathcal{H}_{\text {kin }}=\frac{1}{2 \pi} \oint \boldsymbol{u}^{\prime} \cdot \operatorname{rot} \boldsymbol{u}^{\prime} \mathrm{d} \phi, \quad \mathcal{H}_{\text {curr }}=\frac{1}{2 \pi \mu_{0} \rho} \oint \boldsymbol{B}^{\prime} \cdot \operatorname{rot} \boldsymbol{B}^{\prime} \mathrm{d} \phi$.

Both are zero as long as no instability exists, with proceeding instability and growing nonaxisymmetric modes the helicity quantities also become unequal to zero. An example plot of $\mathcal{H}_{\text {kin }}$ for $R=0.5$ and $z=0.5$ is shown in Fig. 5. After a transition, a steady-state value is reached for both $\mathcal{H}_{\text {kin }}$ and $\mathcal{H}_{\text {curr }}$. The current helicity is approximately 40 times larger than the kinetic helicity in the example shown in the center of the cylinder. In the vertical direction we observed the same behavior as for the fluctuations of flow and magnetic field. Near the top and bottom, the helicities are stronger than those in the region between $z=0.35$ and $z=0.65$. For a positive gradient in angular velocity $\mathcal{H}_{\text {kin }}$ is negative and $\mathcal{H}_{\text {curr }}$ positive. With the changing sign of $\mathrm{d} \Omega / \mathrm{d} z$, the sign of $\mathcal{H}_{\text {kin }}$ and $\mathcal{H}_{\text {curr }}$ also changes, whereas the sign of $B_{z}^{0}$ has no influence. And, as expected, the absolute value of $\mathcal{H}_{\text {kin }}$ is proportional to the squared value of the external field. For Pm $=15$ the relation between $B_{z}^{0}$ and $\mathcal{H}_{\text {kin }}$ is shown in Fig. 6. The fitted parabola does not exactly cross the origin. This shift is attributed to the instability requiring a certain value of $B_{z}^{0}$ (i.e. $B_{z}^{0}=0.04$ for $\mathrm{Pm}=15$ ) to set in with a certain value for $\mathcal{H}_{\text {kin }}$ larger than zero. 

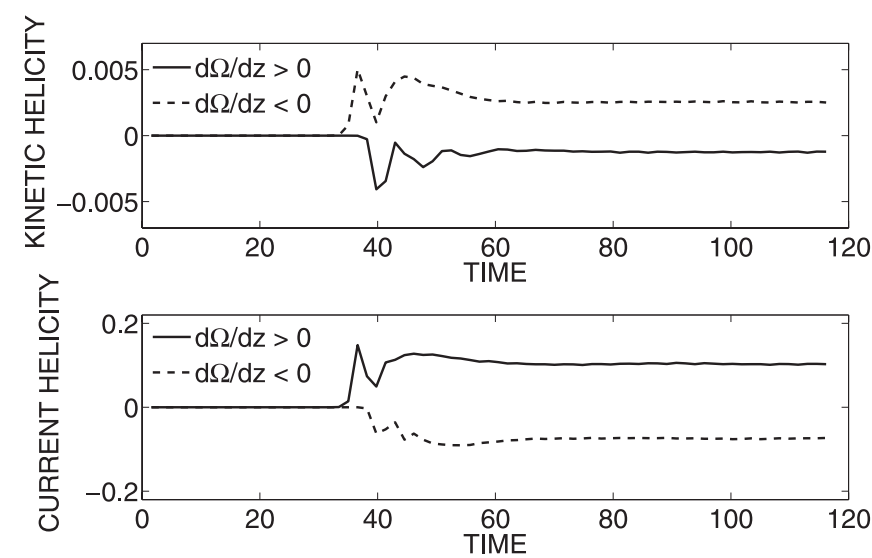

Fig. 5. Kinematic helicity $\mathcal{H}_{\text {kin }}$ and current helicity $\mathcal{H}_{\text {curr }}$ at $R=0.5$ and $z=0.5$ for $\mathrm{Pm}=15$ : both have different signs whereas signs of current helicity and gradient of angular velocity $\mathrm{d} \Omega / \mathrm{d} z$ are the same. The external field values are $B_{z}^{0}=0.05$ for negative and $B_{z}^{0}=0.06$ for positive gradient. Note the different size of $\mathcal{H}_{\text {kin }}$ and $\mathcal{H}_{\text {curr }}$.

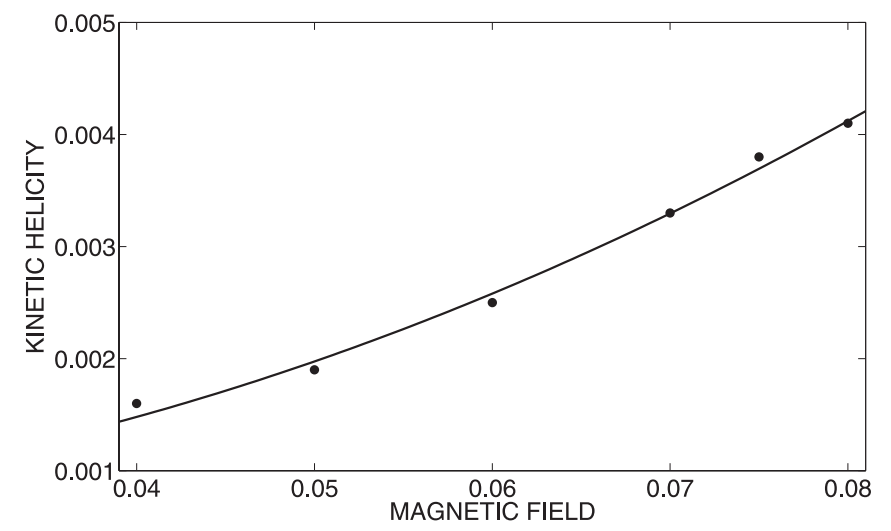

Fig. 6. Kinematic helicity $\mathcal{H}_{\text {kin }}$ as function of the external field $B_{z}^{0}$ with fitted parabola. Values of $\mathcal{H}_{\text {kin }}$ are taken at $R=0.5$ and averaged along vertical direction for $0.35<z<0.65$.

\section{Alpha effect}

Equation (2) suggests that the changing sign of the helicities by changing the direction of $\mathrm{d} \Omega / \mathrm{d} z$, will also appear for the term $\alpha_{z z}$ in the $\alpha$-tensor responsible for the regeneration of the magnetic field. Indeed, calculating $\alpha_{z z}=\left\langle\boldsymbol{u}^{\prime} \times \boldsymbol{B}^{\prime}\right\rangle_{z} / B_{z}^{0}$ using the $z$-component of the electromotive force, gives values for $\alpha_{z z}$ with the same sign as $d \Omega / d z$ for $z<0.65$ (see Fig. 7). Near the top boundary, the disturbances already discussed for the helicities change the values of $\alpha_{z z}$ dramatically to the opposite sign. We excluded this region and took mean values of $\alpha_{z z}$ in the same range $0.35<z<0.65$ used for the helicities and find coefficients between $\alpha_{z z}=0.038$ for $B_{z}^{0}=0.04$ and $\alpha_{z z}=0.067$ for $B_{z}^{0}=0.08$. The correlation time derived from expression (2) using both helicities, $\tau_{\text {corr }}=3 \alpha_{z z} /\left(\mathcal{H}_{\text {curr }}-\mathcal{H}_{\text {kin }}\right)$, is of the order of the system rotation time. Note that the $\alpha$-effect here is mainly due to the current helicity and the product $\alpha_{z z} \mathcal{H}_{\text {curr }}$ is positive. If the external field is switched off, all magnetic field modes decay in the investigated parameter region of low Reynolds numbers and magnetic Prandtl numbers of the order of ten. The helicities drop down to zero nearly immediately. Despite the high magnetic Prandtl number, we do not observe any dynamo action,

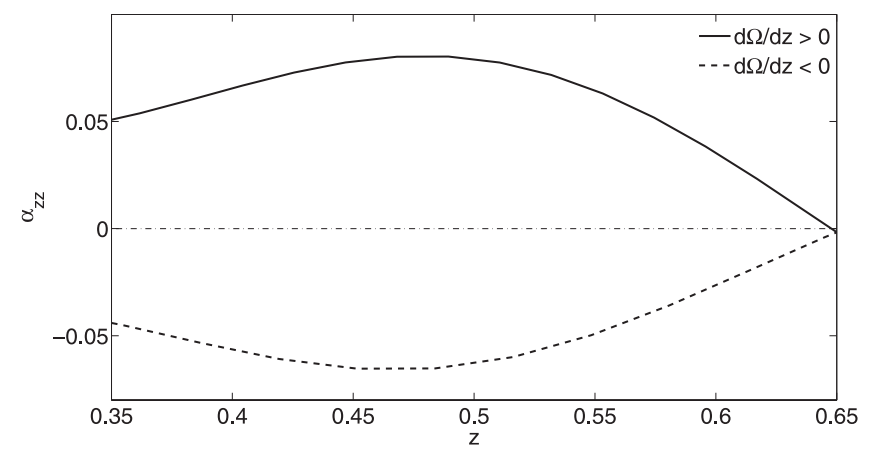

Fig. 7. Coefficient of the $\alpha$-effect $\alpha_{z z}$ at $R=0.5$ for positive and negative $\mathrm{d} \Omega / \mathrm{d} z$. Sign of $\alpha_{z z}$ is the same as $\mathcal{H}_{\text {curr }}$ and $\mathrm{d} \Omega / \mathrm{d} z$.

as found in comparable geometry (Braithwaite 2006). Also, the reported cyclic behavior of the instability did not occur in our simulations.

\section{Conclusions}

We have demonstrated how the instability of toroidal magnetic fields leads to helicity generation in the nonaxisymmetric parts of a flow without density stratification. The suggested relation between helicity, external field, and differential rotation, $\mathcal{H} \propto$ $B_{i}^{0} B_{j}^{0} \Omega_{i, j}$, is rather well fulfilled in the case of $z$-dependent differential rotation. Both the kinematic helicity and current helicity depend on the squared value of the $z$-component of the external magnetic field $B_{z}^{0}$, and scale linearly with the gradient of the differential rotation. Also, the magnetically dominated $\alpha$-effect depends on the direction of $\mathrm{d} \Omega / \mathrm{d} z$, that is $\alpha_{z z}$ holds the same sign as $\mathrm{d} \Omega / \mathrm{d} z$.

The realized model is too simple to estimate consequences of an $\alpha$-effect based on this new kind of helicity production for environments without density stratification and possibly for new dynamo models. In a next step, therefore, we would like to check the relation (4) when differential rotation depending on the distance from the rotation axis is present as well. With an appropriate flow it becomes $\mathcal{H}_{\text {kin }} \propto B_{R}^{0} B_{z}^{0} \mathrm{~d} \Omega / \mathrm{d} R$. In this case, helicity generation should be observable only if both components of the external field are unequal to zero. The problem with this constellation is the changing sign of the product of both field components in one hemisphere in the simplest configuration, a dipolelike field.

\section{References}

Braithwaite, J. 2006, A\&A, 449, 451

Braithwaite, J., \& Spruit, H. C. 2004, Nature, 43, 819

Brandenburg, A., \& Dobler, W. 2002, Comp. Phys. Comm., 147, 471

Brandenburg, A., \& Subramanian, K. 2005, Phys. Rep., 417, 1

Brandenburg, A., \& Subramanian, K. 2007, A\&A, in prep.

Kitchatinov, L. L., \& Rüdiger, G. 2005, Astron. Nachr., 326, 379

Krause, F., \& Rädler, K.-H. 1980, Mean-field magnetohydrodynamics and dynamo theory (Berlin: Akademie-Verlag)

Maeder, A., \& Meynet, G. 2003, A\&A, 411, 543

Pouquet, A., Frisch, U., \& Leorat, J. 1976, JFM, 77, 321

Rüdiger, G., \& Kitchatinov, L. L. 1997, Astron. Nachr., 318, 273

Rüdiger, G., Hollerbach, R., Schultz, M., \& Elstner, D. 2007, MNRAS, accepted

Tayler, R. J. 1973, MNRAS, 161, 365

Vainshtein, S. I., \& Kitchatinov, L. L. 1983, GAFD, 24, 273

Vandakurov, Yu. V. 1972, SvA, 16, 265 